\title{
Méthodologie d'un inventaire de sites de microcentrales Cas de la Haute-Savoie
}

\author{
Methodology for producing an inventory of sites for small \\ hydroelectric schemes. Haute-Savoie, a case study.
}

\author{
G. Degoutte et J. Lavabre
}

CEMAGREF - Groupement d'Aix-en-Provence

Depuis quelques années le problème des "microcentrales " est souvent à l'ordre du jour. Il s'agit selon les uns d'une source d'énergie " nouvelle " particulièrement intéressante, selon les autres, d'un mode d'équipement des rivières particulièrement néfaste.

De nombreuses opérations sont envisagées par des privés ou des collectivités locales. L'approche économique de ces projets parait souvent insuffisante et parfois risquée.

Il importe donc de pouvoir disposer de documents objectifs faisant l'inventaire par région, des sites envisageables, de leur productibilité, leur rentabilité et aussi leurs nuisances.

Le travail présenté ici a été réalisé par le CEMAGREF groupement d'Aix-en-Provence. Il aboutit à un inventaire de sites potentiellement équipables en " microcentrales " au fil de l'eau (gamme de puissance de 200 à $4500 \mathrm{Kw}$ ) avec une estimation des productions électriques, du débit d'équipement optimum et du taux de rentabilité de chaque site. Le département retenu comme application est un département comportant à la fois une zone de montagne et une zone de plaine : la Haute-Savoie. Ce département alpin, ayant a priori de fortes potentialités hydro-électriques, est soumis à différents régimes hydrologiques; c'est pourquoi la méthodologie développée à cette occasion est aisément généralisable.

\section{Méthodologie}

\section{Inventaire sur carte des sites potentiels}

Il s'agit à ce stade du recensement systématique sur cartes IGN au $1 / 25000^{\circ}$ des sites a priori équipables. On calcule la puissance équipée pour un débit d'équipement provisoirement supposé égal au module interannuel. Il n'est pas indispensable d'avoir déjà réalisé l'étude hydrologique complète. Un ordre de grandeur des modules est obtenu en cartographiant les débits spécifiques connus pour toutes les stations limnigraphiques. La puissance est alors, à une constante connue près, le produit $\mathrm{H} \times \mathrm{S}$ :

$\mathrm{H}$ hauteur de chute géométrique

S surface du bassin versant au niveau de la prise.

Lorsqu'on envisage un bassin versant en montagne on place généralement la restitution le plus bas possible, c'est-à-dire à la confluence. Pour la prise il faut étudier plusieurs cas, et l'on cherche à maximiser le produit $\mathrm{H} \times \mathrm{S}$. Bien sûr, sur un tronçon de rivière donné, on se place de préférence à l'aval immédiat d'une confluence ( $A$ est préféré à $\mathrm{A}^{\prime}$ ) ou à l'amont immédiat d'une cascade (figure 1)

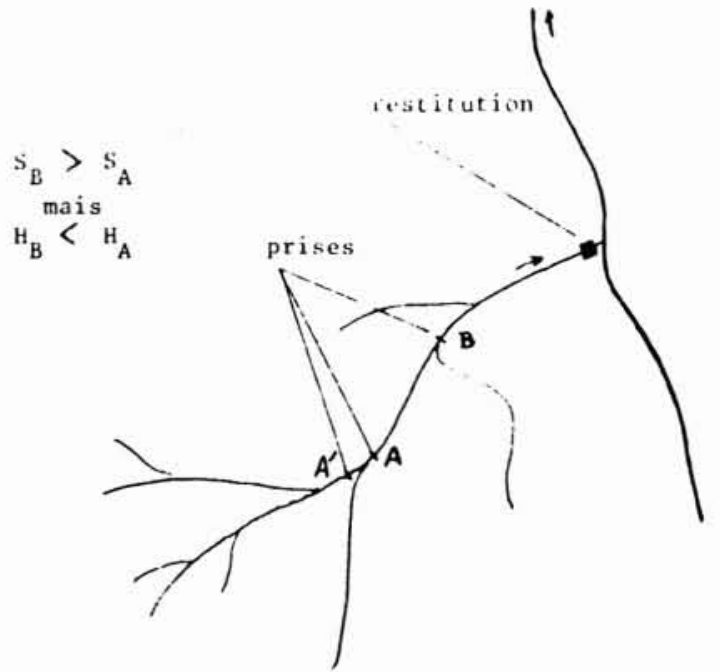

Figure 1 - Choix de l'emplacement de la prise (en montagne). 


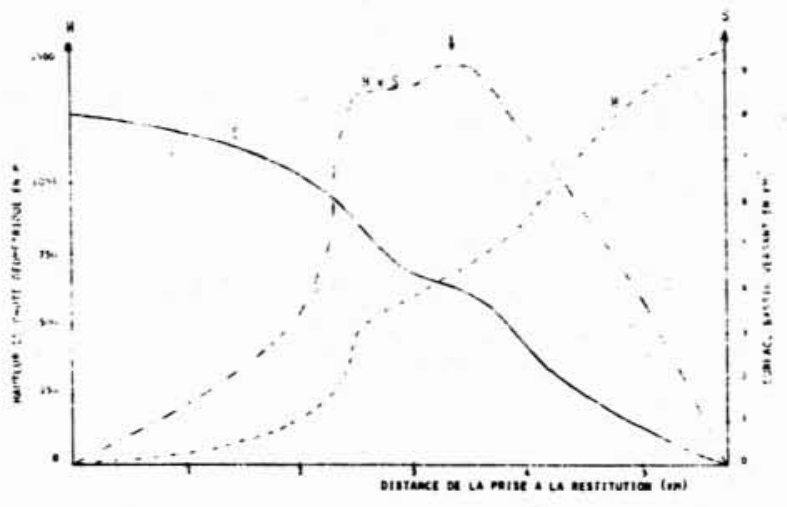

Figure 2 - Optimisation de l'emplacement de la prise.

Pour maximiser $\mathrm{S} \times \mathrm{H}$, il faut procéder par tâtonnement (figure 2).

En plaine, le problème est un peu plus compliqué car l'emplacement de la restitution dépend surtout de considérations locales (routes...)

\section{Etudes hydrologiques, hydrauliques, économiques}

But: Il s'agit de trouver en tout site, les productions électriques annuelles et saisonnières pour plusieurs débits d'équipement. Ces productions tiennent compte des rendements (turbines et génératrices) et d'un débit réservé non turbiné.

La gamme de débits d'équipement testés est fixée par référence au module interannuel du bassin versant QMA; par exemple : QMA/2 à 2 QMA.

En un site a priori équipable, on ne dispose très généralement pas de données de débit. Il est donc nécessaire de travailler sur l'ensemble des données hydrométriques de la région à étudier (département par exemple et bien sûr sa proche périphérie), en vue de les extrapoler aux sites trouvés dans l'inventaire sur carte.

Pour connaître les productions électriques en tout point, on se place dans un premier temps en des sites théoriques que sont les stations limnigraphiques. Là, on calcule les productions moyennes été et hiver pour un mètre de chute. Puis, pour extrapoler en tout point, on cherche des lois donnant ces productions en fonction de paramètres simples. Les paramètres sélectionnés dans le cas étudié sont l'altitude moyenne du bassin versant, le module interannuel QMA et bien sur le débit d'équipement. On s'est alors ramené à la connaissance d'un seul paramètre hydrologique : QMA, qui s'avère être un paramètre intermédiaire particulièrement pratique.

Pour connaitre le QMA en tout point, on recherche une loi donnant sa valeur en fonction de paramètres géographiques et pluviométriques (par exemple altitude et pluviométrie annuelle).

A ce stade, sont connues en tout point les productions saisonnières par mètre de chute, en fonction du débit d'équipement. On se place alors aux sites recensés; une étude économique permet de définir le débit d'équipement optimum et le taux de rentabilité interne du projet.

Les sites peuvent donc être classés en fonction de leur intérêt économique.

\section{Etude hydrologique - Cas de la Haute-Savoie}

Conformément à la méthodologie adoptée, l'étude hydrologique doit donner en tout point du département

- une valeur moyenne du module annuel (QMA) et de sa variation interannuelle. Ceci implique l'établissement d'une loi liant QMA à des paramètres climatologiques et physiques simples du bassin versant;

- une aproche des débits d'étiage pour estimer les débits réservés.

\section{Les données hydrologiques}

Le travail initial a consisté en un recensement le plus large possible des différentes stations limnigraphiques du département et de sa proche périphérie. Nos remerciements vont aux différents organismes gestionnaires (Service Régional Aménagement des Eaux, Direction Technique Générale de E.D.F., Direction Interdépartementale de l'Industrie) qui ont gracieusement mis à notre disposition les séries d'observations en leur possession.

Dans une plage de superficie de bassin versant variable de 10 à $500 \mathrm{~km}^{2}, 47$ stations sont recensées (dont 25 dans la gamme 10 à $100 \mathrm{~km}^{2}$ ). Après la phase de critique des données par des méthodes classiques (double cumuls, écarts cumulés, analyse en composantes principales), 13 stations seront écartées, pour des raisons variées : période d'observation trop courte, redondance avec d'autres stations, séries non homogènes.

Nous disposons finalement de 34 stations dont 11 avec données journalières et 23 avec données mensuelles et couvrant tout ou partie de la période 1960-1980. Après homogénéisation par la méthode des composantes principales, nous avons pu obtenir sur la période 1965-1977 des séries complètes des modules annuels. La carte 3 condense l'information pluviométrique. Elle est établie sur la base de la carte des isohyètes des normales 1936-1965 établies sur le Sud-Est de la France par la D.T.G. Cette carte présente l'avantage de prendre en compte les résultats de pluviomètres totalisateurs situés en altitude. Le tracé des isohyètes est affiné à l'aide des résultats publiés sur la monographie $\mathrm{n}^{\circ} 91$ de la Météorologie Nationale (48 postes sur le département). Malgré la période d'observation différente (1951-1970) il n'y a pas de grande contradiction avec le tracé précédent. Cette constance dans le temps des moyennes pluviométriques indique que la carte ainsi établie est un indicateur pluviométrique robuste susceptible d'interprétation sur la période 1965-1977 pour laquelle nous possédons les séries de modules annuels.

\section{Etude des modules annuels}

La carte 4 présente les valeurs des lames écoulées annuelles moyennes (1965-1977), ainsi que les coefficients de variation de ces séries. La forte variation géographique de ces valeurs dénote la diversité des régimes hydrologiques de ce département alpin : 


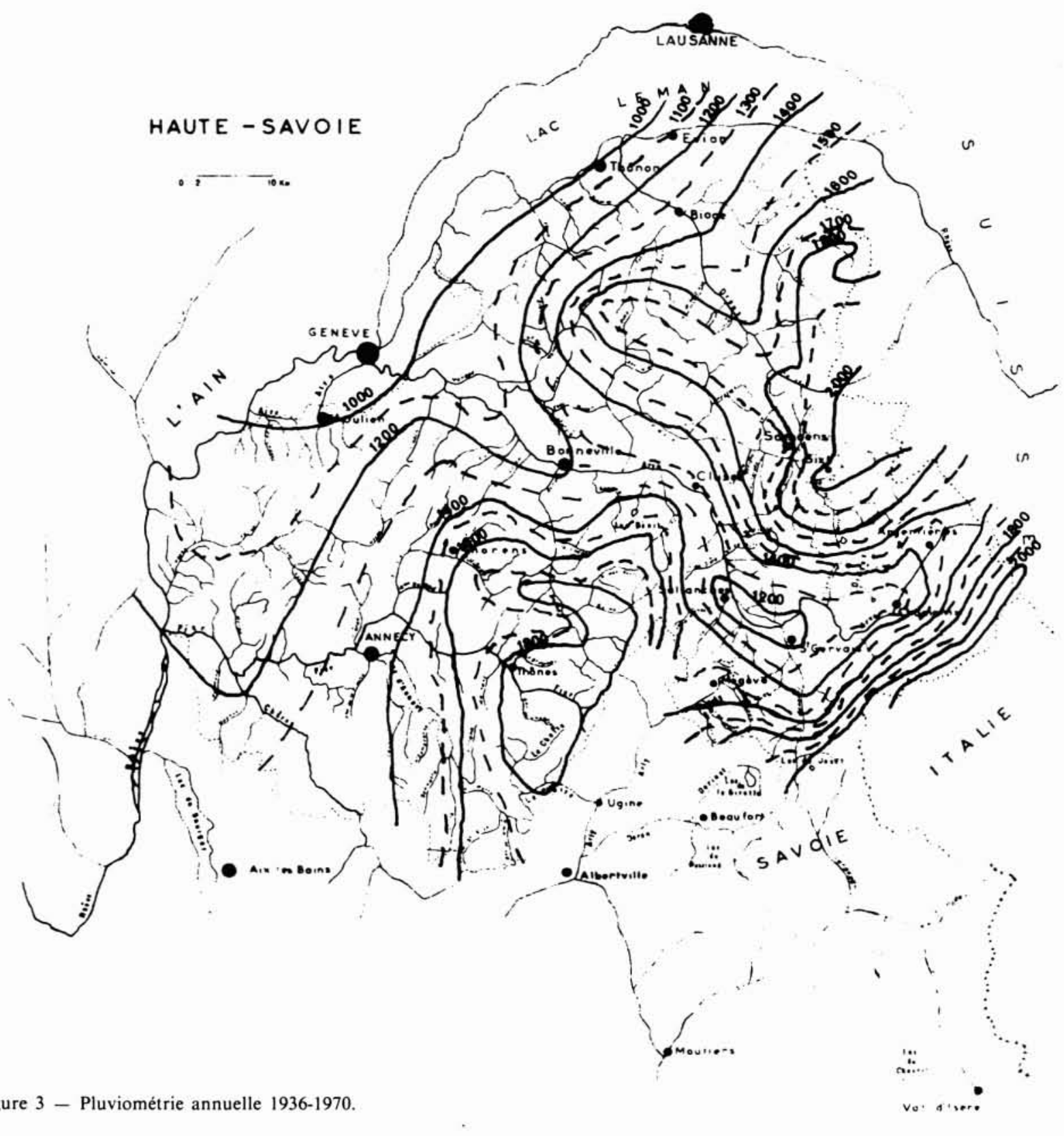

Figure 3 - Pluviométrie annuelle 1936-1970.

$$
\mathrm{QMA}=\frac{S}{31,5}\left(\frac{P}{\beta}\right)^{5 / 3}\left(\frac{z_{m}}{100}\right)^{1 / 3}
$$

- lames avoisinant $2000 \mathrm{~mm}$ pour les torrents glaciaires avec des coefficients de variation faible (de l'ordre de $15 \%$.

- pour la bordure Ouest du département d'altitude plus faible, les QMA sont inférieurs à $700 \mathrm{~mm}$. Inversement les coefficients de variation sont plus élevés (supérieurs à $40 \%$ pour certains bassins),

- entre ces extrêmes on retrouve des valeurs correspondant aux régimes nivoglaciaire, nival, nivopluvial.

Les variables explicatives des lames écoulées sont classiquement la pluviométrie annuelle $P$, la superficie $S$ et l'altitude moyenne $z_{m}$ du bassin versant. La loi type retenue est (1):
$S$ est exprimé en $\mathrm{km}^{2}, \mathrm{P}$ en $\mathrm{mm}, \mathrm{z}_{\mathrm{m}}$ en $\mathrm{m}$ et QMA en $\mathrm{l} / \mathrm{s}$. $\beta$ est un paramètre régionalisé. Il varie dans l'intervalle $(37,46)$. Sa distribution géographique représentée sur la carte ( figure 5) correspond globalement à des zones de végétation homogène, induites par des critères géologiques, climatologiques... Cette équation de régression explique $98 \%$ de la variance, à laquelle correspond un écart-type résiduel de $63 \mathrm{~mm}$.

(1) Référence : Projet de synthèse nationale des apports Informations techniques du CTGREF septembre 1980. 


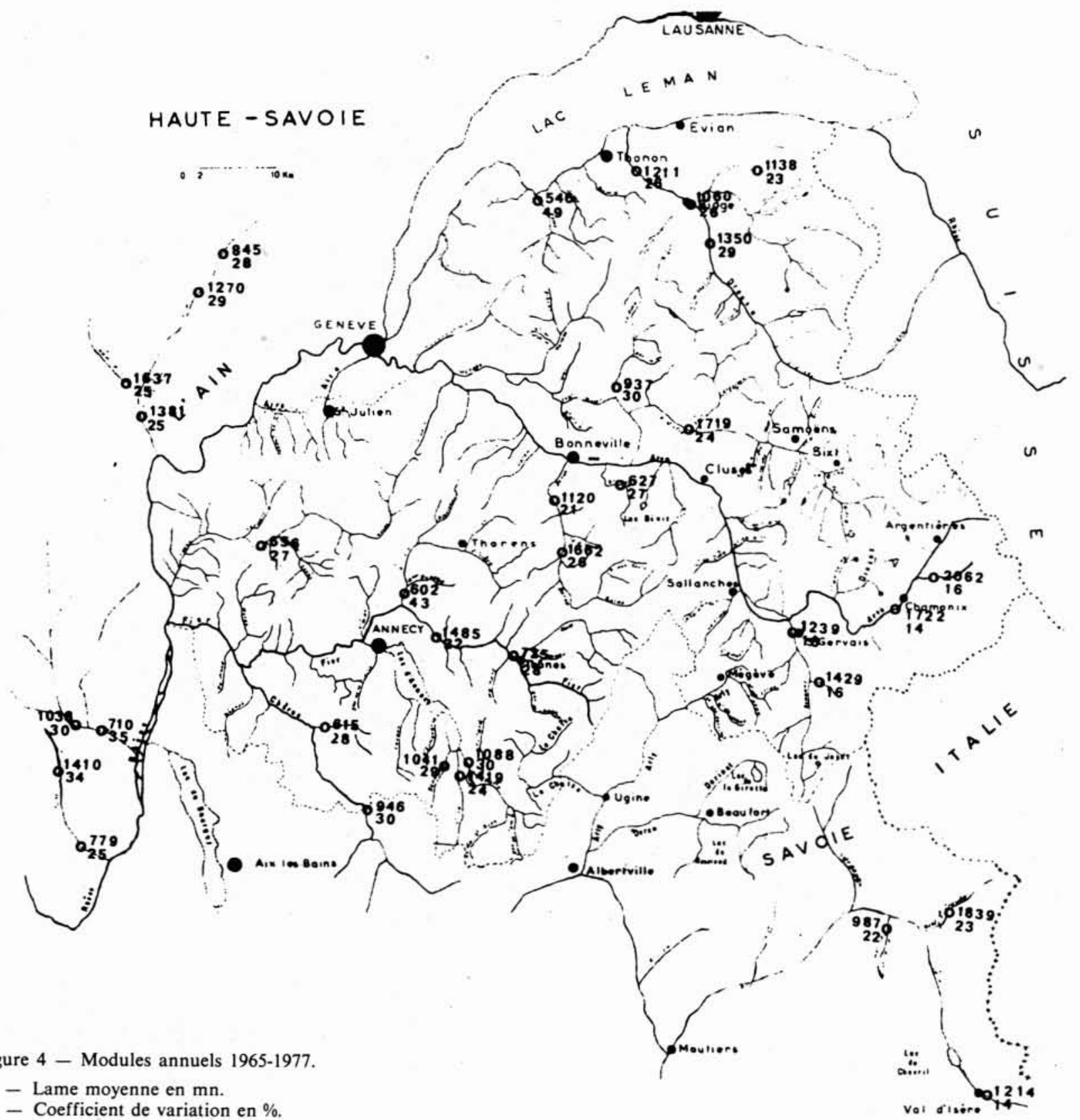

\section{Etude des débits d'étiage}

La fixation du débit réservé est réglementée par des considérations uniquement hydrologiques. L'instruction interministérielle du 15 avril 1981 fixe le débit réservé à la valeur quinquennale du débit caractéristique d'étiage de 10 jours consécutifs $\left(\mathrm{QCN} 10_{5 \text { ans }}\right)$ ou à défaut au débit moyen mensuel minimal (QMS). Actuellement le CEMAGREF travaille selon une approche différente, en introduisant un débit de référence biologique (D.R.B.) qui s'appuie sur des considérations hydrauliques et biologiques caractéristiques de chaque cours d'eau.

Suite à ces remarques et aussi, il faut le dire, à la dispersion des valeurs obtenues pour QMS ou QCN 10, nous ne dégageons pas une loi globale donnant un débit réservé mais une fourchette de valeurs caractéristiques des étiages. Remarquons aussi que les différents régimes hydrologiques conditionnent de manière fondamentalement différente les étiages. En altitude les basses eaux sont observées en hiver (décembre, janvier, février) sur des sols enneigé alors que pour les bassins versants de plaine les étiages correspondent plus conventionnellement au tarissement des nappes et affectent principalement les cours d'eau en été (juillet, août).

Sur les 11 stations pour lesquelles nous disposons des chroniques journalières, les estimations des $\mathrm{QCN} 10_{5 \text { ans }}$ varient dans une fourchette 3 à $81 / \mathrm{s} / \mathrm{km}^{2}$, soit 5 à $15 \%$ du QMA. Soulignons l'influence de l'étiage sévère de 1976 sur certaines de ces valeurs. Une dispersion du même ordre affecte les valeurs du QMS qui est calculé pour 


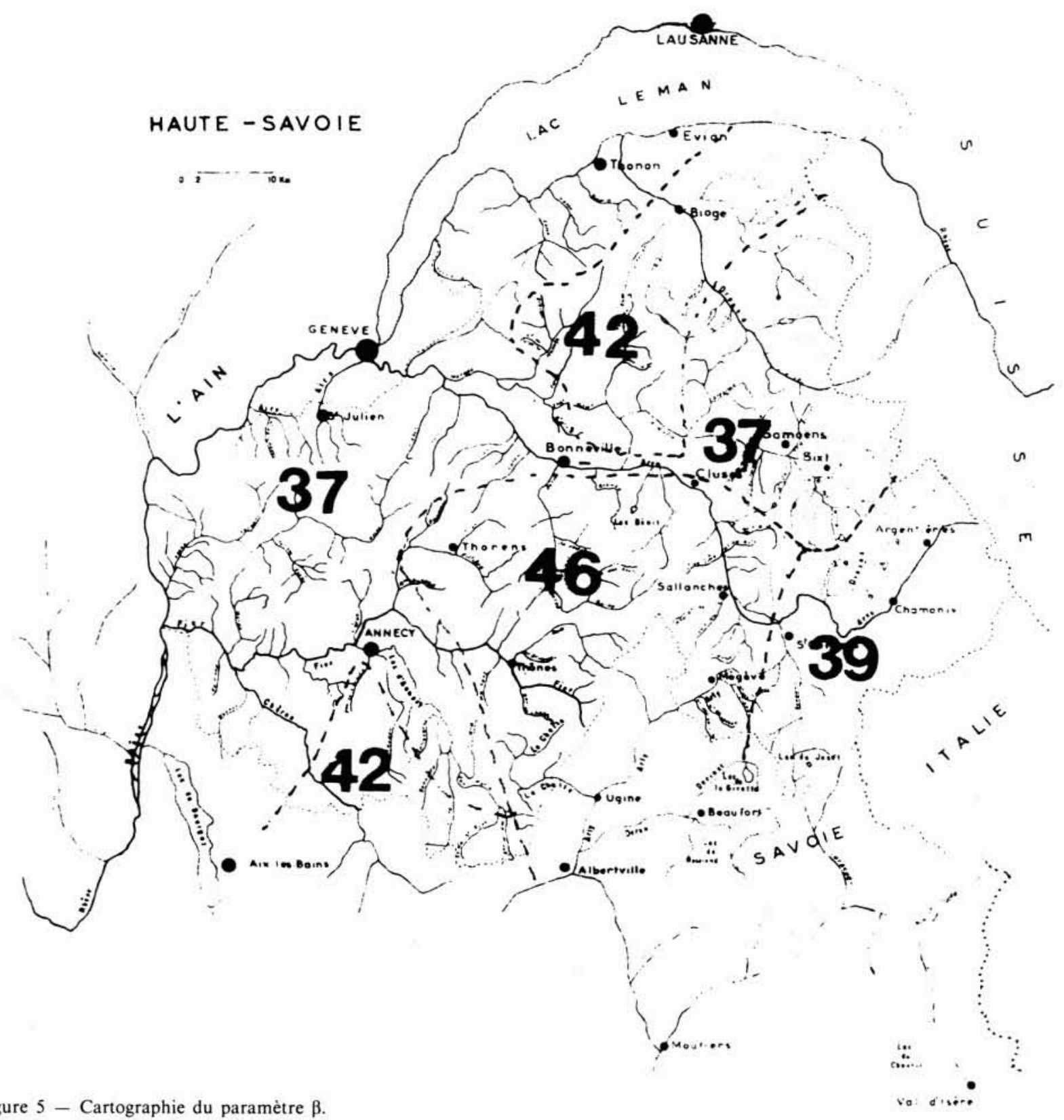

Figure $5-$ Cartographie du paramètre $\beta$.

l'ensemble des stations. La cote 1500 mètres NGF environ sépare les 2 types d'étiage :

- de $600 \mathrm{~m}$ à $1500 \mathrm{~m}$ étiages estivaux, croissance du QMS avec l'altitude dans une plage de 5 à $15 / \mathrm{s} / \mathrm{km}^{2}$ (soit 10 à $35 \%$ du QMA);

- pour des altitudes supérieures, étiages hivernaux, et décroissance du QMS avec l'altitude; il atteint $5 \mathrm{l} / \mathrm{s} / \mathrm{km}^{2}$ pour des altitudes avoisinant $2500 \mathrm{~m}$.

\section{Simulation des productions électriques}

Elle est effectuée sur les stations limnigraphiques que nous appelons sites théoriques. Un logiciel de simulation a été écrit et calcule les productions électriques saisonnières et annuelles pour une hauteur de chute nette de un mètre. Il travaille à partir des données journalières ou mensuelles, teste plusieurs débits d'équipement et plusieurs débits réservés et tient compte des courbes de rendement moyennes de 3 types de turbines (Kaplan, Francis, Pelton). Par contre, on suppose à ce stade qu'il n'y a qu'une turbine.

La gamme de débit d'équipement est exprimée en fonction de $\mathrm{QMA}=\mathrm{k} \times \mathrm{QMA}$, k variant de 0,25 à 5 . La figure 6 représente pour une station la variation en fonction de $\mathrm{k}$, des productions moyennes et de leurs écarts types, calculés aux pas de temps journaliers et mensuels. Pour les débits d'équipement les moins élevés $(k \leqslant 2)$, les productions des différentes turbines sont sensiblement identiques. Pour l'ensemble des sites la variation des 

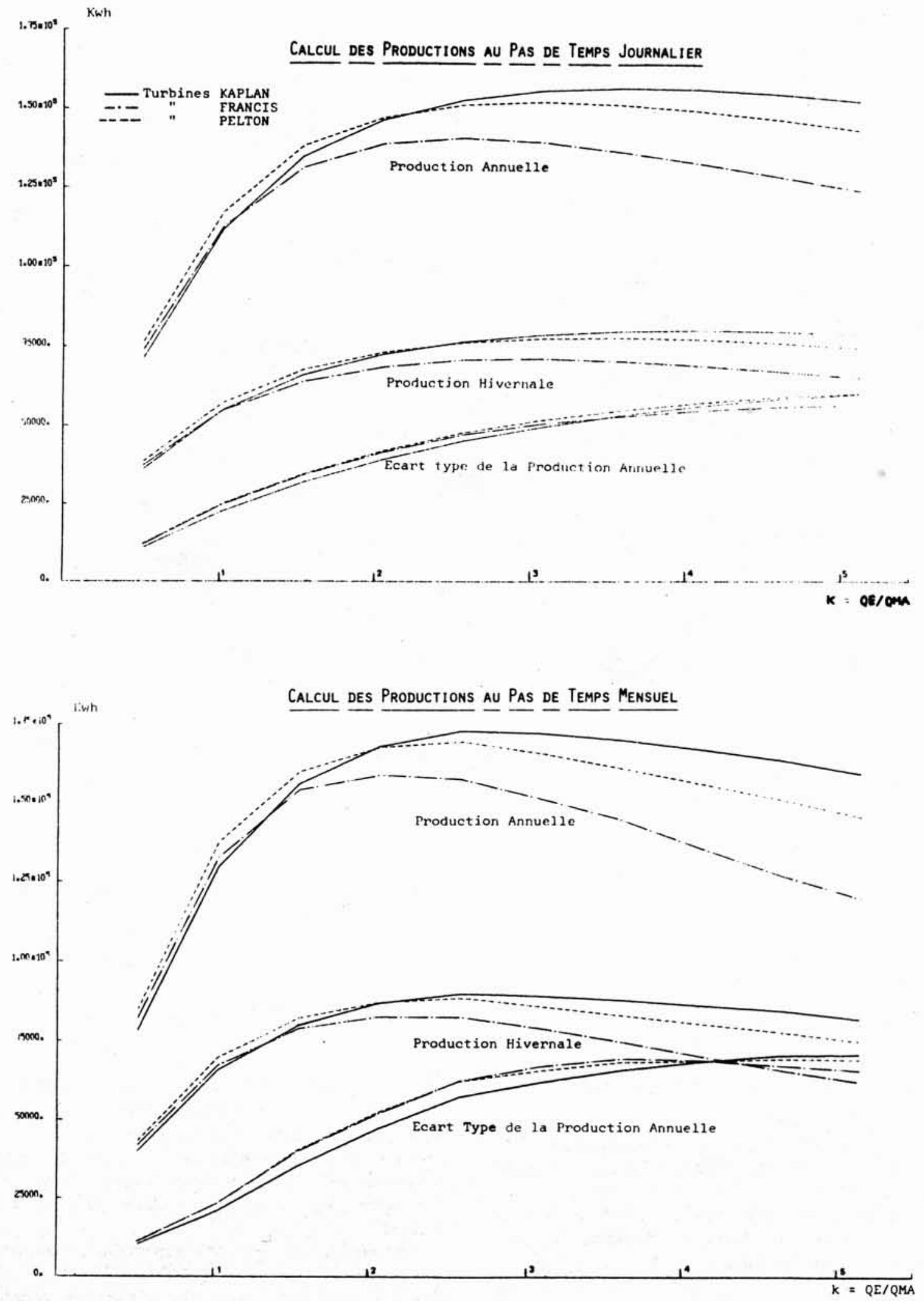

Figure 6 - Station Eaux mortes à Doussard (bassin versant : $\left.92,5 \mathrm{~km}^{2}\right)$. 
productions avec $\mathrm{k}$ présente un optimum qui se situe pour des valeurs de $k$ comprises entre 2 et 4 ; ceci est consécutif au mauvais rendement des turbines qui ne fonctionnent que rarement à leur débit nominal lorsque leur équipement est prévu pour des valeurs de débits élevées. Bien évidemment les écarts types des productions croissent avec k, la variabilité des apports turbinables étant plus grande.

La comparaison des productions calculées au pas de temps journalier PRJ et celles calculées aux pas de temps mensuels PRM fait apparaitre que PRM > PRJ. Ceci est schématisé sur le graphique 7 .

Les résultats de la simulation sont :

- sur 14 stations : productions annuelles et saisonnières simulées au pas de temps journalier (avec $\mathrm{QR}=\mathrm{QCN} \mathrm{10}$ sans $\left._{\text {a }}\right)$ et au pas de temps mensuel $(\mathrm{QR}=0)$; moyenne et écart type de ces productions;

- sur les 23 autres stations, même calcul au pas de temps mensuel seulement.

Pour chaque débit d'équipement testé, des équations de régression de la forme :

$\mathrm{PRJ}=\mathrm{Al} \times \mathrm{PRM}^{\mathrm{BI}}$ (Productions annuelles)

$\mathrm{PHJ}=\mathrm{A} 2 \times \mathrm{PHM}^{\mathrm{B} 2}$ (Productions hivernales)

$\mathrm{SIGJ}=\mathrm{A} 3 \times \mathrm{SIGM}^{\mathrm{B} 3}$ (Écart type des productions annuelles)

sont calées sur les séries des 11 stations pour lesquelles une double simulation a été effectuée. Les coefficients de corrélation sont proches de l'unité : plus de 0,992 pour les productions annuelles, et plus de 0,97 pour les écarts types et les productions hivernales.

Remarquons que par construction ces relations tiennent compte du débit réservé introduit uniquement dans le calcul des productions au pas journalier.

Nous génerons ainsi des productions au pas journaliers sur les 23 stations où l'on n'a utilisé que les chroniques mensuelles.

\section{Loi des productions moyennes annuelles}

Une régression multiple liant les productions PRJ aux différents débits d'équipement $\mathrm{k} \times$ QMA est établie sur 34 stations; $\mathrm{k}$ prend successivement les 6 valeurs : 1/4, 1/2, $3 / 4,1,3 / 2,2$. Elle s'écrit :

soit

$$
\mathrm{PRJ}=29,7 \mathrm{QMA}^{1,043} \mathrm{k}^{0,554}
$$

$$
\mathrm{PRJ}=29,7 \mathrm{QE}^{0,554} \mathrm{QMA}^{0,489}
$$

où

PRJ est exprimée en $10^{3} \mathrm{kWh}, \mathrm{QMA}$ et QE en $\mathrm{m}^{3} / \mathrm{s}$.

Le coefficient de corrélation multiple de cette relation établie sur $34 \times 6=204$ productions est de 0,994 .

Les erreurs de reconstitution: (valeur observée valeur estimée)/valeur estimée $\times 100$ se répartissent comme indiqué sur le tableau ci-contre.

$90 \%$ des reconstitutions sont dans l'intervalle $\pm 20 \%$. Les productions sont sensiblement surestimées sur deux stations pour toute valeur de $k$.

\section{Loi des productions moyennes hivernales}

Tout comme pour les productions annuelles nous avons simulé une série de productions hivernales $\mathrm{PHJ}$ en fonction de différents débits d'équipement. Nous exprimons les productions hivernales en pourcentage des productions annuelles. Ce pourcentage fortement conditionné par les régimes hydrologiques présente une bonne liaison avec l'altitude.

Après établissement de formules de régression pour chaque QE testé, (figure 8), il peut s'écrire sous la forme :

$$
\begin{aligned}
\% \text { hivernal }=\frac{\mathrm{PHJ}}{\mathrm{PRJ}} \times 100= \\
70,4 k^{0,225}-2,24(k-0,2)^{0,640} \frac{z_{m}}{100}
\end{aligned}
$$

valable pour $\quad 500 \mathrm{~m} \leqslant z_{m} \leqslant 2700 \mathrm{~m}$

et

$$
0,25 \leqslant k \leqslant 2
$$
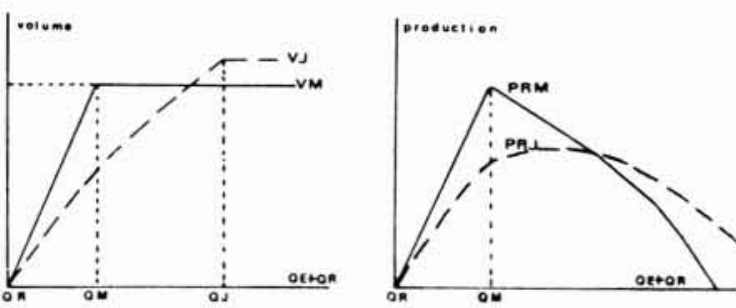

Figure 7 - Comparaison théorique des productions calculées at pas de temps mensuel et au pas de temps journalier.

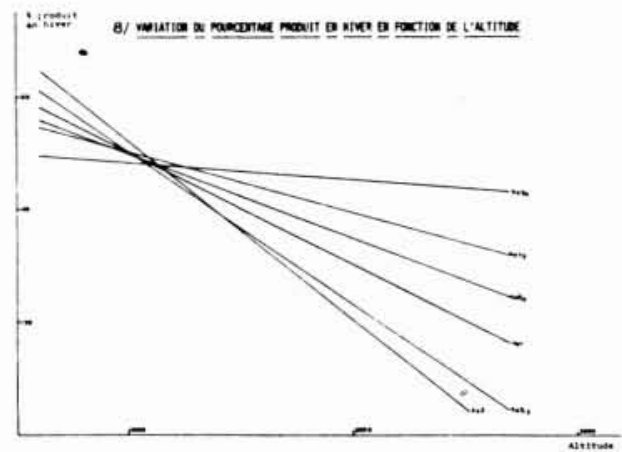

Figure 8 - Variation du pourcentage produit en hiver en fonction de l'altitude.

\begin{tabular}{|c|c|}
\hline $\begin{array}{c}\text { Bornes des erreurs } \\
\text { en } \%\end{array}$ & Pourcentage de cas \\
\hline$-10 \grave{a}+10$ & $63 \%$ \\
\hline$+10 \grave{a}+20$ & $16 \%$ \\
\hline$-10 \grave{a}-20$ & $11 \%$ \\
\hline$+20 \grave{a}+30$ & $2 \%$ \\
\hline$-20 \grave{a}-30$ & $3 \%$ \\
\hline$+30 \grave{a}+40$ & 0 \\
\hline$-30 \grave{a}-40$ & $5 \%$ \\
\hline
\end{tabular}




\section{Loi des écarts types des productions annuelles}

La conduite du calcul économique nécessite la connaissance des fluctuations autour des valeurs moyennes. L'écart type étant un bon indicateur de la variabilité interannuelle nous établissons une formule de régression où les variables explicatives sont le module moyen annuel, le débit d'équipement et l'altitude. Tout comme la moyenne, l'écart type des productions croit avec le QMA et QE; les coefficients de yariation des modules annuels décroissant avec l'altitude (cf. carte 4), il est logique de penser que les écarts types des productions fluctuent de même. On aboutit à la formulation suivante :

$$
\text { SIGJ }=28,0 \mathrm{QMA}^{1,017} k^{0.815}\left(\frac{z_{m}}{100}\right)^{-0,007}
$$

$$
\text { soit } \quad \mathrm{SIGJ}=28,0 \mathrm{QE}^{0,815} \mathrm{QMA}^{0,202}\left(\frac{z_{m}}{100}\right)^{-0,607}
$$

où SIGJ est exprimé en $10^{3} \mathrm{kWh}, \mathrm{QE}$ et $\mathrm{QMA}$ en $\mathrm{m}^{3} / \mathrm{s}$ et $z_{m}$ en mètres.

Le coefficient de corrélation multiple est de 0,966.

Remarquons que le coefficient de variation des productions annuelles qui s'écrit en première approximation :

$$
\mathrm{SIGJ} / \mathrm{PRJ} \simeq 0,94 k^{0,261}\left(\frac{z_{m}}{100}\right)^{-0,607}
$$

est du même ordre que celui des modules annuels. Avec $k=1$, il est de $35 \%$ pour une altitude moyenne de bassin versant de $500 \mathrm{~m}$, et de $15 \%$ pour une altitude voisine de $2000 \mathrm{~m}$ (voir carte 4).

\section{Conclusion pour la simulation des productions}

Ainsi, pour calculer les productions électriques moyennes en un site quelconque il suffit :

- de calculer son module QMA en mesurant le bassin versant, l'altitude moyenne et en calculant la pluviométrie annuelle au prorata des surfaces à partir de la carte 3 ; - de calculer la production annuelle pour plusieurs débits d'équipement à partir de QMA uniquement;

- de calculer la part de production hivernale pour les mêmes débits d'équipement à partir de l'altitude moyenne du bassin versant.

\section{Étude économique des sites inventoriés}

A ce stade on dispose :

- d'une liste de sites a priori envisageables;

- des productions été et hiver pour divers débits d'équipement et pour 1 mètre de chute nette.

\section{Calcul des recettes}

On calcule les productions moyennes saisonnières en multipliant les productions unitaires par la hauteur de chute nette (celle-ci est calculée pour le débit maximal, ce qui est un peu pessimiste pour les périodes de marche à débit partiel).

Les recettes sont calculées, au niveau de l'inventaire, en supposant que l'on a affaire à un producteur autonome livrant toute sa production à E.D.F. et bénéficiant du tarif simplifié 2 prix. Il n'a pas été pris en compte de bonification saisonnière (ce qui est légèrement pessimiste en site de plaine). Par contre, on a pris en compte une majoration de qualité de $10 \%$ pour l'été, et $6 \%$ pour l'hiver; ces valeurs pouvant être atteintes facilement en sites de montagne, où la régularité interannuelle est bonne.

Ces trois hypothèses simplificatrices (pertes de charges pour le débit maximum, bonifications et primes) sont largement admissibles au niveau d'un inventaire ou d'une étude de faisabilité.

Plusieurs hypothèses d'évolution des tarifs sont envisagées, mais avec une augmentation du rapport prix d'hiver/prix d'été.

\section{Calcul des charges d'exploitation}

Elles varient sensiblement selon les types de maitres d'ouvrage. On a retenu l'hypothèse moyenne d'une charge annuelle égale à $3 \%$ de l'investissement.

\section{Méthode de calcul}

La méthode retenue est celle du taux de rentabilité interne calculé pour une durée de vie supposée égale à 30 ans.

On calcule, pour chaque débit d'équipement, l'investissement, les charges d'exploitation, les recettes (en francs constants). Pour chacun des débits d'équipement testés, on obtient alors un taux de rentabilité interne.

Le débit d'équipement retenu pour le site est celui qui conduit au meilleur taux de rentabilité interne. On peut alors classer les sites par rentabilité décroissante. Dans le cas particulier de l'étude de Haute-Savoie, on a considéré qu'un taux de rentabilité interne inférieur à $3 \%$ conduisait à éliminer le site. Au-delà, les sites sont jugés dignes d'une étude de faisabilité. Le meilleur site a un taux de rentabilité de $15 \%$ (bien entendu, ces chiffres sont relatifs aux hypothèses retenues : durée de vie, évolution des tarifs...). Les débits d'équipement optimum varient dans la fourchette 0,25 QMA à QMA.

Enfin, et cela sort du cadre de la présente note, une première approche des conditions d'environnement a été menée. Elle conduit à écarter un certain nombre de projets intéressants au plan économique, pour des raisons de site ou de qualité piscicole du cours d'eau à dériver.

\section{Conclusion}

Le recensement des sites envisageables de même que les études économiques de chaque site sont des opérations relativement classiques. Par contre, l'approche poursuivie pour l'étude hydrologique est relativement intéressante. Elle permet, en effet, de réaliser en tout site, une première étude de faisabilité. Il suffit de disposer de la hauteur de chute, de la surface du bassin versant et de son altitude pour calculer :

- le débit moyen interannuel;

- les productions moyennes été et hiver;

- les écarts types de ces variables.

A côté de la synthèse nationale des apports et de celle des débits de crue des petits bassins versants, un tel travail est l'amorce d'une synthèse nationale des productibles au fil de l'eau. 